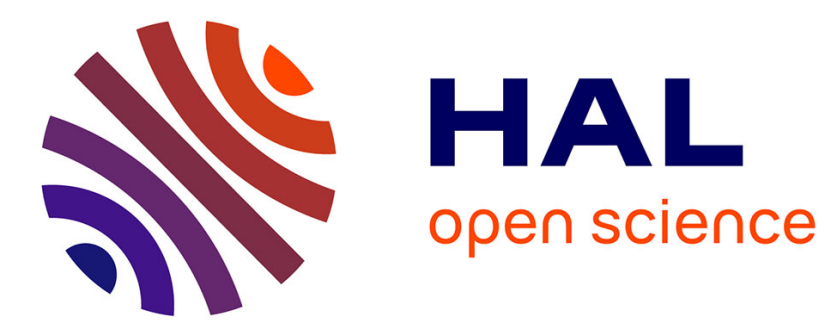

\title{
Influence de la structure sur l'effet hall des lames minces de nickel phosphore
}

\author{
J. Fléchon, M. Viard
}

\section{To cite this version:}

J. Fléchon, M. Viard. Influence de la structure sur l'effet hall des lames minces de nickel phosphore. Revue de Physique Appliquée, 1970, 5 (5), pp.765-769. 10.1051/rphysap:0197000505076500 . jpa00243452

\section{HAL Id: jpa-00243452 https://hal.science/jpa-00243452}

Submitted on 1 Jan 1970

HAL is a multi-disciplinary open access archive for the deposit and dissemination of scientific research documents, whether they are published or not. The documents may come from teaching and research institutions in France or abroad, or from public or private research centers.
L'archive ouverte pluridisciplinaire HAL, est destinée au dépôt et à la diffusion de documents scientifiques de niveau recherche, publiés ou non, émanant des établissements d'enseignement et de recherche français ou étrangers, des laboratoires publics ou privés. 


\title{
INFLUENCE DE LA STRUCTURE SUR L'EFFET HALL DES LAMES MINCES DE NICKEL PHOSPHORE
}

\author{
par J. FLÉCHON et M. VIARD \\ Laboratoire de Physique de dépôts métalliques \\ Faculté des Sciences de Nancy
}

(Reçu le 21 mai 1970)

\begin{abstract}
Résumé. - L'effet Hall des lames minces de nickel-phosphore préparées par oxydo-réduction (1-2) à partir de volumes égaux d'oxydant et de réducteur, est fortement influencé par la température de préparation $\left(0^{\circ} \mathrm{C}, 20^{\circ} \mathrm{C}, 50^{\circ} \mathrm{C}\right)$. La conductivité est électronique. Les résultats vérifient convenablement les théories actuelles de l'effet Hall des ferromagnétiques.
\end{abstract}

\begin{abstract}
The Hall effect of thin deposits of phosphor-nickel obtained by oxydo-reduction for equal volumes of oxidant and reducer, is strongly influenced by the temperature of the bath $\left(0^{\circ} \mathrm{C}, 20^{\circ} \mathrm{C}, 50^{\circ} \mathrm{C}\right)$. The conductivity is of electronic character. The results obtained are in keeping with the prevailing theories.
\end{abstract}

Etude expérimentale. - Les conditions d'étude des lames minces de nickel-phosphore préparées par oxydo-réduction en phase liquide à $0^{\circ} \mathrm{C}$ et $50^{\circ} \mathrm{C}$ sont identiques à celles définies à $20^{\circ} \mathrm{C}$ dans de précédentes communications [3-4-5]. Le dosage de ces dépôts [1-2] permet de mettre en évidence, pour une épaisseur déterminée, une concentration en phosphore plus forte à $0{ }^{\circ} \mathrm{C}$ et $50^{\circ} \mathrm{C}$ qu'à $20^{\circ} \mathrm{C}$. Soit pour une lame de $700 \AA$ :

Température de prépara-

tion .............. $\quad 0^{\circ} \mathrm{C} \quad 20^{\circ} \mathrm{C} \quad 50^{\circ} \mathrm{C}$

Lame de $700 \AA$ de type. A $\quad$ B $\quad$ C

P $\%$ en poids........ $9,8 \% \quad 7,5 \% \quad 8 \%$

Mesures de la tension de Hall. - L'ensemble des mesures porte sur 40 lames de chaque type dont l'épaisseur varie de $350 \AA$ à $2500 \AA$.

Pour chacune d'elles, nous avons étudié l'influence $\mathrm{du}$ recuit sous vide à diverses températures : $100^{\circ} \mathrm{C}$, $200^{\circ} \mathrm{C}, 300^{\circ} \mathrm{C}, 400^{\circ} \mathrm{C}$ (les temps correspondent à une stabilisation de la résistance [2]) et, pour un recuit donné, nous avons mesuré la tension de Hall $V_{\mathrm{H}}$ pour différentes températures (de $20^{\circ} \mathrm{C}$ à $350^{\circ} \mathrm{C}$ pour des films recuits à $400^{\circ} \mathrm{C}$ ).

Une étude aux rayons $\mathrm{X}$, effectuée sur des échantillons épais de type A, B, C, nous permet de caractériser la structure du dépôt considéré.

Les figures 1 et 2 représentent les diagrammes $\mathrm{X}$ d'une lame $\mathrm{A}$ recuite respectivement à $200^{\circ} \mathrm{C}$ et $400^{\circ} \mathrm{C}$. Elle mettent en évidence l'existence de deux structures différentes. Une phase amorphe caractérisant un dépôt recuit entre $20^{\circ} \mathrm{C}$ et $200^{\circ} \mathrm{C}$ que nous appellerons état homogène, et une structure cristalline (après recuit au-delà de $250^{\circ} \mathrm{C}$ ) avec apparition d'une phase nickel (raies 111, 200, 220) et d'une phase

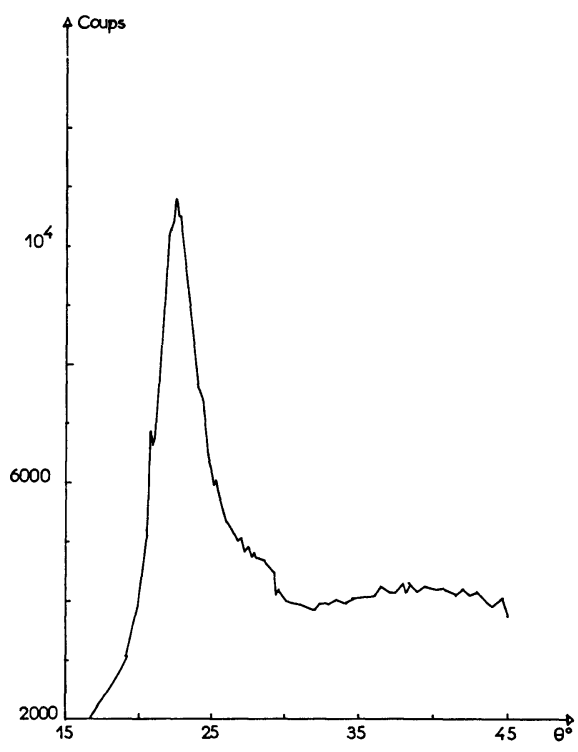

Fig. 1. - Diagramme X. Recuit $200^{\circ} \mathrm{C}, \quad 11 \%$ de $\quad$ P.

phosphure de nickel $\mathrm{Ni}_{3} \mathrm{P}$. Celles-ci étant entièrement précipitées après recuit à $400^{\circ} \mathrm{C}$ : état précipité. Deux éléments étroitement liés semblent conditionner l'effet Hall ; la concentration en phosphore : effet de composition; l'état cristallin associé à la nature des phases et à la grosseur des grains composant la matrice : effet de structure. L'influence de chacun d'eux peut être examinée dans la mesure où l'on s'efforce de maintenir l'autre fixe dans des échantillons différents.

Dans l'état homogène, la figure 3 montre l'importance de la température de préparation des lames sur la tension de Hall $V_{\mathrm{H}}$ mesurée à $20^{\circ} \mathrm{C}$ sous vide classique; l'effet de structure l'emporte sur l'effet de 


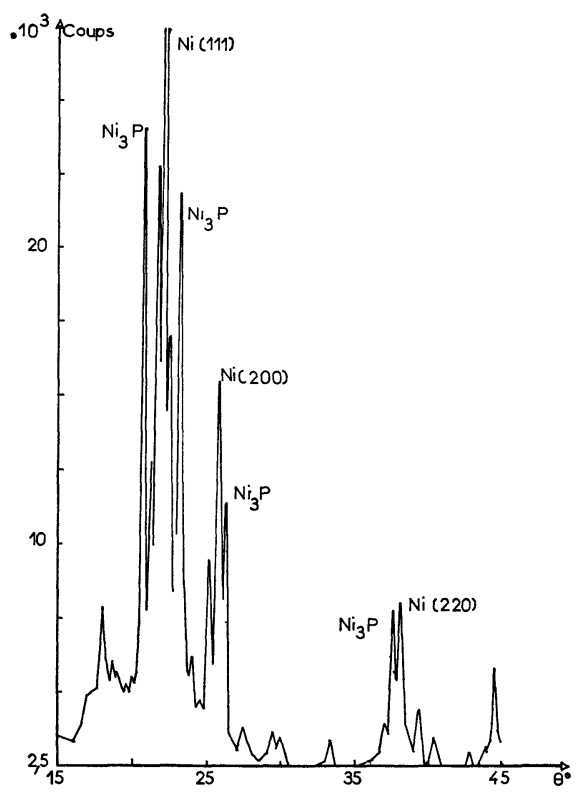

FIG. 2. - Diagramme X. Recuit $400^{\circ} \mathrm{C}, 11 \%$ de P.

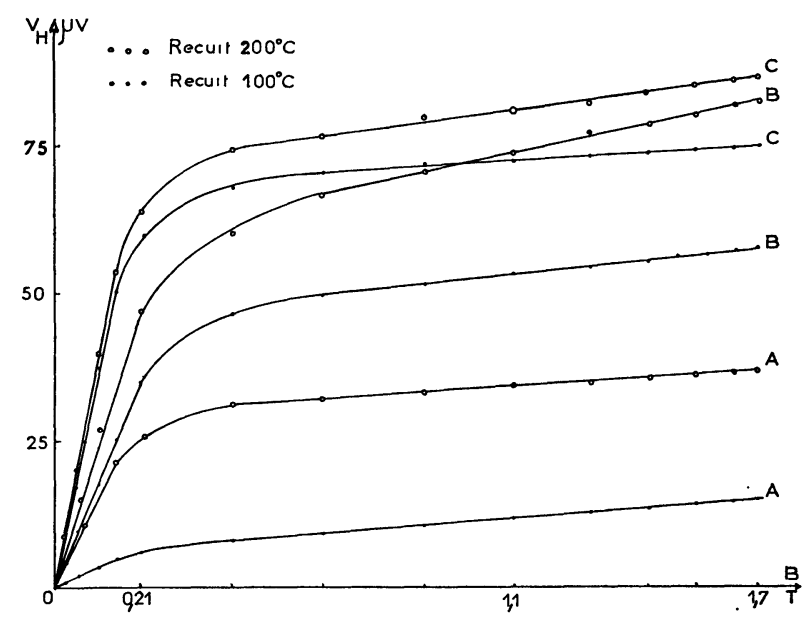

FIG. 3. $-V_{\mathrm{H}}=f(B)$ en phase homogène.

composition, les raies $\mathrm{X}$ des dépôts ainsi obtenus s'affinent et s'intensifient lorsque la température du bain s'élève. De même, après une stabilisation sous vide à $100^{\circ} \mathrm{C}, V_{\mathrm{H}}$ croît de $\mathrm{A}$ à $\mathrm{C}$.

Sur la même figure, le recuit à $200^{\circ} \mathrm{C}$ se traduit pour chaque type de lame par une augmentation importante de $V_{\mathrm{H}}$. Une diffusion d'atomes de phosphore avec apparition de liaisons nickel-phosphore et de microcristaux de phosphure de nickel, pourrait être à l'origine de ce phénomène. La concentration en phosphore va évidemment influer sur le caractère ferromagnétique du dépôt. Fléchon [1] l'a observé sur des poudres obtenues de la même manière. La saturation est moins prononcée dans le cas de la lame $\mathrm{A}$ la plus riche en phosphore et la moins bien organisée.

Des mesures magnétiques récentes effectuées par Maéda [7] sur des films de nickel-phosphore électro- lytique, montent le rôle important joué par le phosphore comme inclusion non magnétique.

Dans l'état précipité, les mesures de $V_{\mathbf{H}}$ faites dans les mêmes conditions et sur les mêmes lames, accusent une chute importante, en même temps que s'affirme le caractère ferromagnétique (précipitation du nickel). Résultat qui confirme les mesures absolues d'aimantation de Fléchon [1].

Les diagrammes $\mathrm{X}$ comportent des raies du phosphure de nickel $\mathrm{Ni}_{3} \mathrm{P}$ d'autant plus intenses qu'augmente le taux de phosphore présent dans l'alliage. Les valeurs de $V_{\mathrm{H}}$ semblent liées à la présence de $\mathrm{Ni}_{3} \mathrm{P}$; les plus faibles étant observées sur des lames de nickel pur de même épaisseur recuites dans les mêmes conditions [11]. Le phosphore engagé dans la combinaison $\mathrm{Ni}_{3} \mathrm{P}$ donne à $400^{\circ} \mathrm{C}$ des pics de maximum plus élevé pour $\mathrm{C}$ que pour $\mathrm{A}$, correspondant à des grains plus gros [7] : l'effet de structure l'emporte sur l'effet de composition.

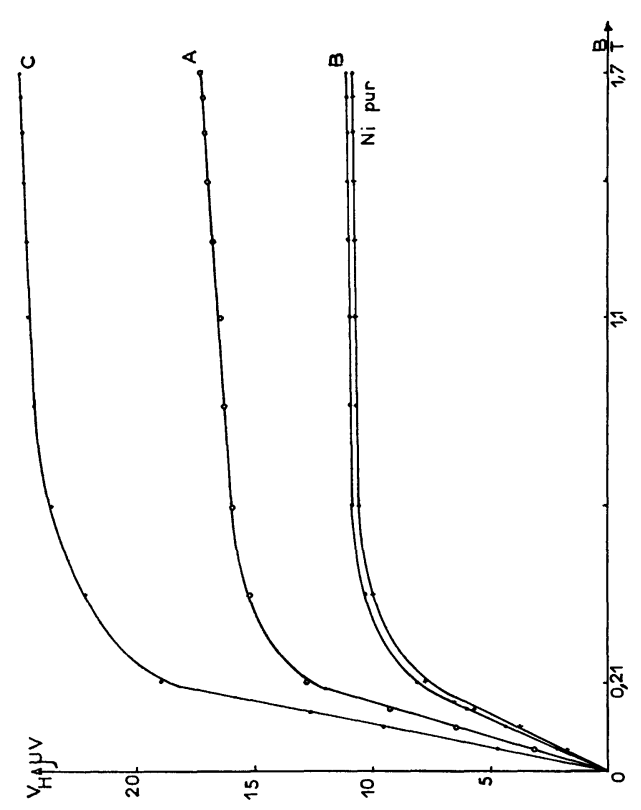

FIG. 4. $-V_{\mathrm{H}}=f(B)$ après recuit à $400^{\circ} \mathrm{C}$.

Les variations de la tension de Hall en fonction de la température, sont identiques à celles rapportées dans des communications antérieures [3-5-6].

Relations entre les résultats expérimentaux et les théories actuelles de l'effet Hall des ferromagnétiques. - Pugh [8] a donné une loi expérimentale concernant la résistivité de Hall $\rho_{\mathrm{H}}$ qui s'écrit dans le système international :

$$
\rho_{\mathrm{H}}=\mu_{0}\left(R_{0} \mathrm{H}+R_{1} J\right)=R_{0} \mathrm{~B}+\mu_{0} R_{\mathrm{s}} J
$$

$R_{0}=$ constante de Hall ordinaire en $\mathrm{m}^{3} / \mathrm{C}$.

$R_{1}=$ constante extraordinaire en $\mathrm{m}^{3} / \mathrm{C}$.

$R_{\mathrm{s}}=$ constante de Hall spontanée en $\mathrm{m}^{3} / \mathrm{C}$.

$J=$ aimantation en $\mathrm{A} / \mathrm{m}$. 
La figure 5 montre que dans le cas des lames A, la résistivité de Hall croît régulièrement avec l'épaisseur.

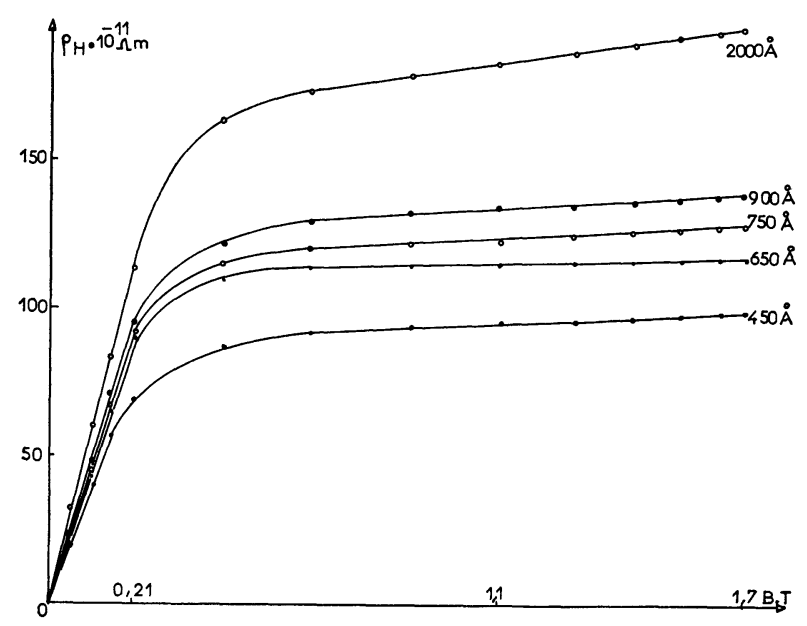

FIG. 5. $-P_{\mathrm{H}}=f(B)$ lames recuites à $400^{\circ} \mathrm{C}$.
Ce phénomène est nouveau si on le compare à celui qui caractérise les lames $B$ et $C$ précédemment étudiées [4-5] pour lesquelles la croissance ne s'observait qu'entre $300 \AA$ et $550 \AA$. L'explication de ce comportement est naturelle si l'on observe que la lame A contient sur toute son épaisseur un taux de phosphore comparable à celui qui caractérise $\mathrm{B}$ et $\mathrm{C}$ de 0 à $550 \AA$ A.

La constante ordinaire $R_{0}$, liée à la conductivité électrique est négative. Les valeurs de $R_{0}$ obtenues sur l'ensemble des échantillons épais, nous permettent de déduire le nombre $n$ d'électrons de conduction par atome [9-10]:

$$
n=-\frac{1}{R_{0} \cdot N \cdot e}
$$

$N=$ nombre d'atomes par unité de volume. $e=$ charge de l'électron.

Les valeurs de $R_{0}$ et n sont données par le tableau I.

TABLEAU I

\begin{tabular}{|c|c|c|c|c|c|}
\hline \multicolumn{3}{|c|}{ Echantillon } & A & B & $\mathrm{C}$ \\
\hline \multirow{4}{*}{$\begin{array}{c}\text { Etat } \\
\text { homogène }\end{array}$} & Recuit & $R_{0} \times 10^{-11} \mathrm{~m}^{3} / \mathrm{C}$ & $-\overline{33}$ & $-\overrightarrow{62}$ & -38 \\
\hline & $100^{\circ} \mathrm{C}$ & $n^{e-} /$ atome & 0,21 & 0,12 & 0,18 \\
\hline & Recuit & $R_{0} \times 10^{-11} \mathrm{~m}^{3} / \mathrm{C}$ & -20 & -44 & -36 \\
\hline & $200^{\circ} \mathrm{C}$ & $n^{e-} /$ atome & 0,24 & 0,16 & 0,20 \\
\hline \multirow{3}{*}{$\begin{array}{c}\text { Etat } \\
\text { précipité }\end{array}$} & Recuit & $R_{0} \times 10^{-11} \mathrm{~m}^{3} / \mathrm{C}$ & $-25,5$ & -15 & -20 \\
\hline & $400^{\circ} \mathrm{C}$ & $n^{e-} /$ atome & 0,27 & 0,47 & 0,37 \\
\hline & $\left.\begin{array}{l}\text { Nickel pur [11] } \\
\text { Recuit à } 400^{\circ} \mathrm{C}\end{array}\right\}$ & $R_{0}=-6 \times 10^{-11}$ & $; n=1,14$ & & \\
\hline
\end{tabular}

Nous remarquons que pour chaque type de lame, $\mathrm{n}$ croît avec la température de recuit, ce phénomène pourrait être lié à l'ordre qui se crée dans le réseau.

Dans l'état homogène, nous pouvons considérer comme Maéda [7] et Albert et Kovac [12], que le matériau est une solution saturée de phosphore dans le nickel. Le phosphore libre participant à la conductivité, $\mathrm{n}$ croît avec le taux de soluté.

Pour interpréter les valeurs de $R_{0}$ et $n$ du nickel et de ses alliages, Beitel et Pugh [10] ont imaginé un modèle à quatre bandes de conduction. Deux bandes sphériques $4 \mathrm{~s}$ dont les électrons $n_{\mathrm{sp}}$ ont leur moment de spin parallèle au champ d'induction $B$ et $n_{\mathrm{sa}}$ à moment antiparallèle au champ, $n_{\mathrm{sp}}+n_{\mathrm{sa}}=n_{\mathrm{s}}=0,54$ et deux bandes sphériques $3 d$ à $n_{d \mathrm{p}}$ et $n_{d \mathrm{a}}$ trous. Dans l'état homogène, $n$ est inférieur à $n_{s} / 2$. Dans certaines conditions, l'effet Hall des pseudo-nickels $(\mathrm{Ni}, \mathrm{Cu}$, $\mathrm{Fe})$ [13-14] conduit à des valeurs de même ordre. Les auteurs considèrent alors que dans ce cas, seul $n_{\mathrm{sp}}$ contribue à l'effet Hall.

Le passage de l'état homogène à l'état précipité est marqué par une nette augmentation de $\mathrm{n}$ qui demeure cependant inférieur à 1,14 valeur caractérisant le nickel pur [11]. Contrairement à l'observation relative à l'état homogène, $n$ décroît d'autant que le taux de phosphore présent dans le matériau est grand. L'interprétation qualitative de cette propriété, semble liée à la formation du phosphure de nickel $\mathrm{Ni}_{3} \mathrm{P}$ qui mobilise d'autant plus d'atomes de nickel que le taux de phosphore est important. Dans des conditions analogues, Ehrlich et Dreesen [13] après avoir étudié l'influence d'un métal étranger $(\mathrm{Fe}, \mathrm{Co})$ sur l'effet Hall des lames de nickel, ont constaté une variation du même ordre et ont conclu que ce phénomène était indépendant de la nature de l'impureté incluse dans la phase. Nous le vérifions dans le cas du phosphore.

Dans cet état, l'application du modèle de bandes donné par Pugh, conduit à considérer que $n_{d}=0,5$ trous de la bande $3 d$ participent à la conduction [6]. Pugh et Rostoker, lors d'une étude d'effet Hall sur des alliages nickel-cuivre, ont trouvé $n_{\mathrm{s}}=n_{d}=0,6$.

La constante extraordinaire $R_{1}$ est liée au ferromagnétisme du matériau; dans le cas du nickel-phosphore elle est négative. Une faible valeur absolue de $R_{1}$, correspond donc à un caractère ferromagnétique plus prononcé. Nous remarquons en effet que $\left|R_{1}\right|$ 
décroît au fur et à mesure des recuits et particulièrement lorsque le nickel précipite. Ce phénomène a été mis en évidence par Fléchon [1] dans la détermination $\mathrm{du}$ coefficient d'aimantation à saturation.

Dans les deux cas considérés précèdemment, $\left|R_{1}\right|$ croît avec le taux de phosphore inclus dans la lame, comme l'indique la figure 6 où nous avons représenté la variation de $\left|R_{1}\right|$ en fonction de la température de mesure. Les valeurs de la constante ayant été obtenues à partir de lames de $2000 \AA$ recuites à $400^{\circ} \mathrm{C}$, prises dans les différents matériaux $\mathrm{A}(11 \%$ de $\mathrm{P}), \mathrm{B}$ $(7,5 \%$ de $\mathrm{P}), \mathrm{C}(8,5 \%$ de $\mathrm{P})$, nickel pur [11]. Ces

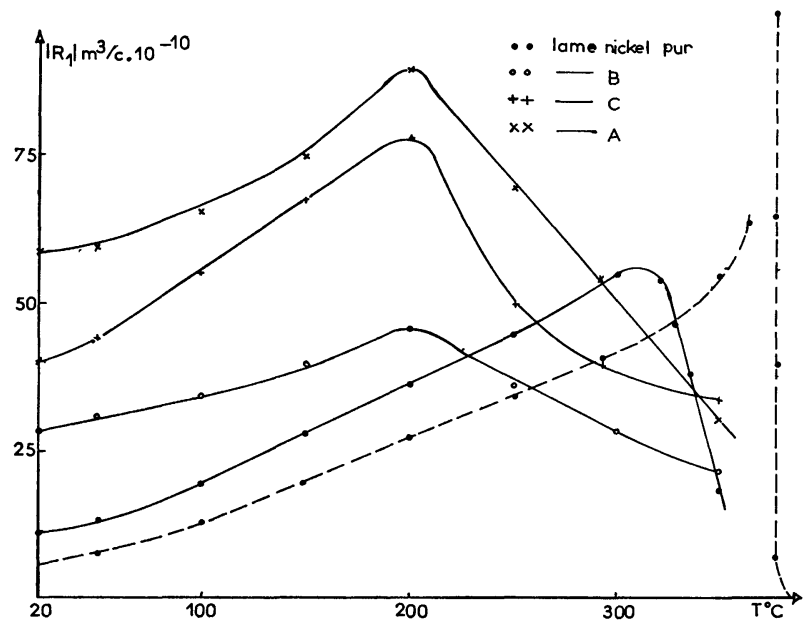

Fig. 6. $-R_{1} \mid=f(T)$ lames recuites à $400{ }^{\circ} \mathrm{C}$.

observations sont en accord avec les mesures de Alber et Kovac [12] qui exprime le moment magnétique $\mu_{B}$ (magnétons de Bohr) d'un alliage de nickel-phosphore en fonction de $n_{\mathrm{o}}=$ nombre de trous de la bande $3 d$ du nickel, $c=$ concentration en phosphore, $Z=$ valence de l'élément par la relation :

$$
\mu_{B}=n_{\mathrm{o}}-c Z
$$

La loi $R_{\mathrm{s}}=\mathrm{A} \rho_{e}^{m} \quad\left(R_{\mathrm{s}}=\right.$ constante de Hall spontanée ; $\rho_{e}$ résistivité électrique) [4] prévue par la théorie de l'effet Hall des ferromagnétiques [15-16] avec $1,4<m<2$ est vérifiée dans le cas du nickel-phosphore. Nous trouvons en effet pour les lames précédentes :

$\begin{array}{ccc}\text { A } & \text { B } & \text { C } \\ 1,40<m<1,70 & 1,50<m<1,70 & 1,45<m<1,80\end{array}$

Des valeurs analogues ont été obtenues à partir des lames de nickel-fer [17].

Le tracé des courbes $\rho_{\mathrm{H}}$ en fonction de l'induction $B$, nous permet de calculer une valeur approchée de la susceptibilité à l'origine $\chi_{0}$ [18]. Les résultats obtenus à partir de lames $\mathrm{A}, \mathrm{B}, \mathrm{C}$, de $2000 \AA$ recuites à $400{ }^{\circ} \mathrm{C}$ sont reproduits sur la figure 7 et comparés aux valeurs de $\chi_{0}$ données par Don Kirkham [19] pour le nickel pur. A $20^{\circ} \mathrm{C}$, Pascal [20] indique $\chi_{0}=21$ pour le nickel pur. $\chi_{0}$ diminue lorsque le taux de phosphore augmente dans la lame. Nous constatons comme Albert et Kovac [12], Mc Guire, Croll et Koretsky [21] que le point de Curie de l'alliage est plus bas que celui $\mathrm{du}$ nickel pur.

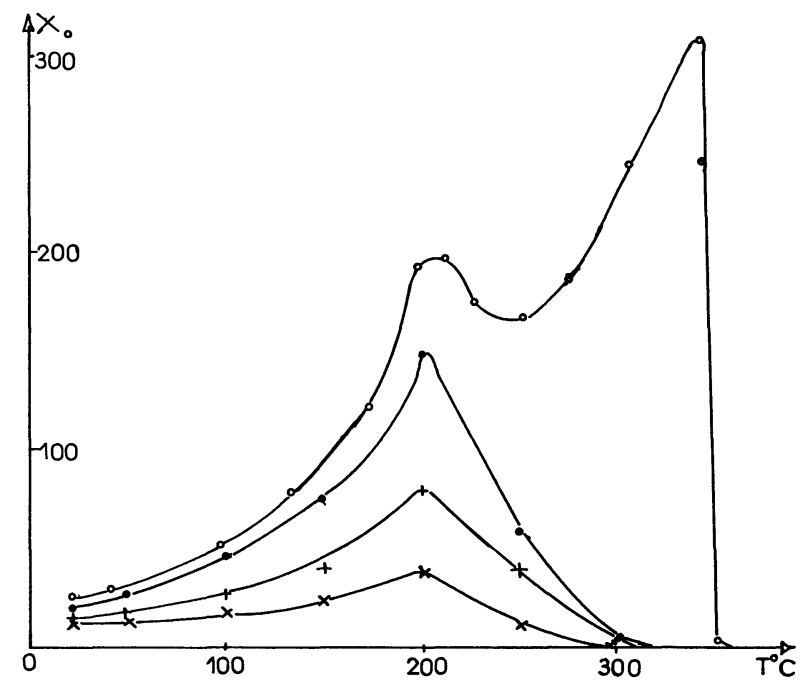

Fig. 7.

- Nickel pur.

- Nickel phosphore réparé à $20^{\circ} \mathrm{C}-7 \%$ de $\mathrm{P}$. $+\quad-\quad-\quad 50{ }^{\circ} \mathrm{C}-9 \%$ de $\mathrm{P}$ $\times \quad-\quad-\quad Q^{\circ} \mathrm{C}-11 \%$ de $\mathrm{P}$.

L'effet Hall des films minces de nickel-phosphore et les grandeurs qui s'y rattachent sont nettement perturbés par une augmentation du taux en phosphore avec un effet de structure important. Les mesures magnétiques de Albert et Kovac [12] sur le nickelphosphore électrolytique, montrent la nécessité d'effectuer une analyse aux rayons $\mathrm{X}$ pour déterminer la nature et la structure des phases en présence. Ces auteurs proposent un modèle où $60 \%$ des atomes de phosphore réagissent avec le nickel pur pour former $\mathrm{Ni}_{3} \mathrm{P}$, le reste demeurant en solution solide. Actuellement, aucune expérience précise ne permet d'affirmer que du phosphore reste en solution après recuit à $400^{\circ} \mathrm{C}$. Nous envisageons une étude au diffractomètre $\mathrm{X}$ à l'aide d'un tube à foyer fin qui permettrait de déterminer avec précision la position des raies du nickel : la présence de phosphore libre devant produire leur déplacement sans que prédomine l'effet de structure. D'autre part, pour une température de préparation donnée, il est possible de modifier le taux du phosphore présent dans la lame sans jouer sur la structure en faisant varier le potentiel rédox du bain.

Cela nous permettra dans une prochaine étude, d'examiner l'influence sur l'effet Hall du pourcentage en phosphore et préciser encore l'effet de composition mis en évidence ici. 


\section{Bibliographie}

[1] FléCHON (J.), Thèse Nancy, 1960.

[2] Fléchon (J.) et Machizaud (F.), C. R. Acad. Sci., 1966, 263, 500.

[3] Fléchon (J.) et Viard (M.), J. Physique, 1968, 29, 937.

[4] Fléchon (J.) et Viard (M.), J. Physique, 1968, 29, C. $2,83$.

[5] Fléchon (J.) et Viard (M.), C. R. Acad. Sci., 1968, $260,1279$.

[6] Fléchon (J.) et Viard (M.), C. R. Acad. Sci., 1970, $270,85$.

[7] MaÉda (H.), J. Appl. Phys. Japan, 1969, 8, 978.

[8] Pugh (E. M.), Phys. Rev., 1930, 36, 503.

[9] Foner (S.) et Pugh (E. M.), Phys. Rev., 1953, 91, 4. Sondheimer (E. H.), Proc. Roy. Soc. London, 1948, A 193, 484.

[10] Beitel (F.) et Pugh (E. M.), Phys. Rev., 1958, 112, 5, 1516.

[11] Goureaux (G.), Thèse Caen, 1960.
[12] Albert (P. A.) et Kovac (Z.), Lilienthal (H. R.), Phys. Rev., 1967, 38.

[13] Ehrlich (A. C.) et Dreesen (J. A.), Phys. Rev., 1964, 133, 408.

[14] Sandford (E. R.), Ehrich (A. C.) et Pugh (E. M.), Phys. Rev., 1961, 123, 1949.

[15] Karpluss (R.) et Luttinger (J. H.), Phys. Rev., 1954, 95, 1154.

[16] Sмiт (J.), Physica, 1955, 21, 877.

SMit (J.), Nuovo Cimento, 1957, 6, 1177.

[17] Rivier (D.) et Huguenin (R.), Helv. Phys. Acta, 1961, 34, 771.

[18] Coren (C.) et Juretschke (H. J.), Phys. Rev., 1957, $28,806$.

[19] Don Kirkham, Phys. Rev., 1937, 1, 1166.

[20] Pascal, Chimie Minérale, 1963, 17, 599.

[21] Mc Guire (T. R.), Croll (I. M.), Koretsky (H.), Bull. Am. Phys. Soc., 1964, 9, 435. 